\title{
Connectivity Reliability and Topological Controllability of Infrastructure Networks: A Comparative Assessment
}

\author{
Jian Li1 ${ }^{1,2,3, *}$, Leonardo Dueñas-Osorio ${ }^{3}$, Changkun $\mathrm{Chen}^{2}$, Congling Shi ${ }^{1}$ \\ ${ }^{1}$ Beijing Key Laboratory of Metro Fire and Passenger Transportation Safety, China Academy of Safety \\ Science and Technology, Beijing 100012, P. R. China \\ ${ }^{2}$ Institute of Disaster Prevention Science and Safety Technology, Central South University, Changsha, \\ Hunan 410075, P. R. China \\ ${ }^{3}$ Department of Civil and Environmental Engineering, Rice University, Houston, TX 77005, U. S. A
}

\begin{abstract}
As infrastructure systems evolve, their design, maintenance, and optimal performance require mature tools from system reliability theory, as well as principles to handle emerging system features, such as controllability. This paper conducts a comparative study of the connectivity reliability (CR) and topological controllability (TC) of infrastructure systems in terms of three aspects: topology, robustness, and node importance. Taking eight city-level power transmission networks and thousands of artificial networks as examples, this paper reveals that a dense and homogeneous network topology is better to satisfy CR and TC requirements, than more common sparse and heterogeneous networks when node attributes are generic. It is observed that the average degree's impact on CR is more significant than on TC, while degree heterogeneity is more significant on TC. When node attributes are accounted for, for generators the reliability-based node importance measure may underestimate some important nodes in terms of TC, and vice versa - an issue not observed for substation nodes. The findings in this paper suggest a potential new direction to enhance reliability-based design by integrating it with emerging controllability-based measures relevant in the future as infrastructure networks increase reliance on information systems.
\end{abstract}

Keywords: Infrastructure networks, connectivity reliability, topological controllability, link density, degree heterogeneity, robustness, node importance

\footnotetext{
* Corresponding author at: Beijing Key Laboratory of Metro Fire and Passenger Transportation Safety, China Academy of Safety Science and Technology, Beijing 100012, P. R. China.

Tel.: +86 135-4965-3773

E-mail: lijianethan@gmail.com (J. Li), leonardo.duenas-osorio@ rice.edu (L. Dueñas-Osorio), cckchen@csu.edu.cn (C. Chen), shicl@ chinasafety.ac.cn (C. Shi)
} 


\section{Introduction}

Networked infrastructure systems (e.g., water, power, telecommunication and transportation), are of critical importance to modern societies. Because of their networked nature, once one component is damaged by a natural, internal or deliberate hazard, other components may malfunction as well, which naturally requires studying their system-level reliability against disruptions [1]. The reliability of infrastructure system refers to the ability of the system to provide adequate services to its customers [2]. System reliability studies show that connectivity reliability (CR) is used as a necessary condition for more sophisticated function-based reliability. Together, these concepts have enabled infrastructure engineering research and implementation to mature and be used in practical reliability-based design [3-5], reliability-based maintenance and management [6], and reliability-based restoration and optimization [7,8] among others.

As infrastructure systems evolve and rely on information technologies more intensively, understanding their controllability [9] also becomes essential. In controllability theory, a system is controllable if it can be driven from any initial state to any desired final state within finite time with a suitable choice of inputs [9]. In this paper, we argue that traditional reliability approaches need to be complemented with controllability management principles to be able to design and manage future smart infrastructure systems. Systematic comparisons are necessary between reliability and controllability features of infrastructure systems so as to understand how the two approaches differ (both at the network level and the element level), or can be used complementarily.

Network CR analyses are typically performed by repeated computational simulations of network connectivity given samples of hazard realizations or failure sequences [10-12]; typical approaches rely on various Monte Carlo simulation strategies, including Markov Chain Monte Carlo [13], Subset Simulation [14], among the others [15]. Some advanced sampling methods are also applied, such as importance sampling [16] and Latin hypercube sampling (LHS) [17,18]. These simulation-based approaches allow for straightforward applications of deterministic network analysis algorithms to a wide class of lifeline network problems, including disconnections and functional requirements. However, the sampling nature of simulation-based approaches may require a large number of simulations to achieve an acceptable level of convergence in the results, especially for low-probability events and large scale systems [19]. 
As an alternative, researchers are also developing various non-simulation-based approaches, including the Disjoint Product Technique (DPT) [20], Binary-Decision Diagrams (BDD) [21] and their improved versions as Ordered Binary Decision Diagrams (OBDD) [22], the Dotson algorithm [23], Recursive Decomposition Algorithms (RDA) [24], Matrix-based System Reliability (MSR) methods [25], universal generating function methods [26], Combinatorial Recursive Methods [27], Linear programming approaches [28], sequential compounding method (SCM) [29,30], and algorithmic enumeration [31] among others, where analytical insight is sought and guaranteed approximations or bounds are also unraveled as their computational complexity tends to be high.

Alternatively, fuzzy logic, uncertainty theory, and imprecise probability methods are also advancing. For instance, Feng et al. [32] presented an imprecise system reliability method based on survival signature, while He and Zhang [33] conducted a fuzzy reliability analysis using cellular automata for network systems, and Hosseini and Wadbro [34] employ uncertainty theory to dispense with the use of probability distributions or fuzzy membership functions. In recent years, scholars have also developed reliability models aiming to specific systems, such as multistage systems $[35,36]$ and correlated failures systems $[37,38]$ to name a few.

Among the mentioned methods, RDA is one of the most transparent in network reliability computation. At its core, RDA identifies the shortest path from a source element to a sink element in a network (or graph), and proceeds with a recursive decomposition by using the Boolean logic and associated probabilistic operations to quantify cut set likelihoods (i.e., joint failures of network components that cause them to be disconnected) and link set probabilities (i.e., joint survivals of components that ensure the connection of the pair). Liu and Li [39,40] proposed RDA efficiency improvements based on network reduction approaches. However, RDA still suffers from the curse of dimensionality for large-size networks, because the numbers of disjoint cut sets and link sets increase exponentially with network size. As an alternative, Lim and Song [19] and Lim et al. [41] proposed a Selective RDA (S-RDA), which identifies the most reliable paths, i.e. critical disjoint cut sets and link sets (and thus a set of bounds) that have dominant contributions to the likelihood of network connection or disconnection. The S-RDA thus searches a smaller state space and can obtain narrow bounds on the failure probability efficiently. Hence, this paper computes network CR via S-RDA, as described in Section 2.1. 
The combinatorial nature of system reliability is also present in the emerging notion of topological controllability (TC) of complex networks, as it integrates classical control theory and network science, anticipating future smart networked systems. Lombardi and Hornquist [42] applied the linear system controllability principles to networks inspired by biology. A node is in this context controllable if an external signal can be applied which can adjust node properties in finite time to an arbitrary value, regardless of the levels of the other nodes. However, these ideas are not directly applicable to large scale networks because of computation complexity. Alternatively, Liu et al. [9] developed analytical tools to identify the set of driver nodes (by finding a maximum matching of a bipartite graph version of the original system) that can in principle guide or control the system's entire dynamics. Liu's algorithm identifies minimum driver node sets even for large scale networks. However, many configurations admit the minimum driver nodes set, among which some nodes are always the driver nodes. These nodes are called critical nodes. There are also some nodes that sometimes are driver nodes, and thus defined as intermittent nodes. The rest of the nodes are called redundant nodes. More recently, Jia et al [43] developed an analytical framework to identify the category of each node, identifying two distinct control modes in complex networks: centralized versus distributed control.

As for the engineering applications of TC principles, though Diao and Rauch [44] have introduced TC theory into infrastructure system (water distribution system as a pre-selection method for sensor placement), it is still necessary to explore how the TC of infrastructure networks relates to their CR which typically guides infrastructure design and upkeep. Also, the role of network topology on CR- and TC-based performance is unclear now. To design and manage infrastructure systems, identifying critical components is important, and whether CRbased approaches match or mismatch with TC-based approaches should be unraveled. Component importance is also key to understand how systems behave under random failures or target attacks [45].

Metrics to evaluate and quantify CR and TC constitute the core of the comparative study. Some TC-based metrics have been developed and applied in the network science community. For example, Yan et al. [46] developed a metric in terms of control cost, which captures the energy needed to control networks. The Control Robustness Index proposed by Wang et al. [47] is a metric to assess controllability against failure and attacks. The minimal number of driver 
nodes is widely used for quantifying the TC attributes of networks [9]. However, the cardinality of the minimal driver node set fails to compare controllability properties among different network scales. As an alternative, in this paper the authors develop a new way to quantify TC, named the Controllability Index (CI), which not only eliminates the impact from network scale, but also identifies the system TC attributes fairly.

As for the node importance measures in terms of TC, a few metrics are proposed. Among them, monitoring the frequency that a node participates in all minimal driver node sets or control backbone is common [48]. But this metric may result in importance ties for some of the nodes, because some are always driver nodes (which are called critical nodes subsequently) and some nodes are never driver nodes (which are called redundant nodes). Another node importance metric is called Control Centrality [49], which quantifies the ability of a single node to control a directed weighted network, although it is not suitable for general unweighted networks. This paper proposes a metric for quantifying the node importance that relies on removing a node, and monitoring changes on $\mathrm{CI}$ and on the number of minimal control schemes. The proposed metric could capture a node's contribution to the system controllability, while minimizing importance tie levels including among unweighted networks.

With the proposed metrics, this study conducts a comparative investigation of infrastructure networks connectivity reliability (CR) and topological controllability (TC) features at both network and node levels. At the network level, this paper not only discusses how link density and degree heterogeneity affect both CR- and TC- based system performance, but also conducts a comparative analysis on CR- and TC- based system robustness characteristics. At the node level, this study discusses similarities and differences between CR- and TC-based node importance measures.

The rest of this paper is structured as follows: in Section 2, this paper provides an overview of S-RDA for CR assessment, and proposes a CR-based node importance (NI) measure. Section 3 presents TC theory and develops a NI measure in terms of TC indicators. Section 4 detects how link density and degree heterogeneity impact both CR and TC features. Section 5 provides a robustness analysis based on both $\mathrm{CR}$ and TC measurements. Node importance in terms of CR and TC is quantified and compared in Section 6. Finally, Section 7 provides general conclusions and ideas for future research. 


\section{Network connectivity reliability (CR) assessment}

Depending on how to decompose a network, the original RDA can be categorized as pathbased RDA [24] and cut-based RDA [40]. The former is suitable for networks with high reliability nodes (or edges), while the latter is more efficient for networks with low reliability nodes (or edges). This paper adopts a path-based S-RDA, since the node reliability in this paper's networks is relatively high, to reflect engineered systems designs.

\subsection{Selective Recursive Decomposition Algorithm (S-RDA)}

The original RDA considers a network consisting of a source node, a terminal node, and other transmission nodes and edges between them, which are represented by a graph $G=(N, A)$, where $N$ and $A$ denote the set of nodes and edges in the graph, respectively. After a damaging event (e.g. natural hazards or system failures), each component (node or edge) is typically modeled with binary states of survival or failure.

The S-RDA takes the most reliable paths as the decomposition policy of the algorithm, and decomposes sub-graphs selectively based on the probabilities of the events indicated by the associated set coefficients found during decomposition. In Lim and Song's paper [19], the SRDA approach for CR between a source node and a terminal node can be computed as:

$$
R=P\left(E_{01}\right)+\sum_{j=1}^{n_{L .1}} P\left(B_{1 j} \cap E_{1 j}\right)+\sum_{j=1}^{n_{L, 2}} P\left(B_{2 j} \cap E_{2 j}\right)+\cdots+\sum_{j=1}^{n_{L, m}} P\left(B_{m j} \cap E_{m j}\right)=\sum_{i=1}^{N_{L}} P\left(L_{i}\right),
$$

where $E_{i j}$ is the event describing the survival of the $j$-th most reliable path identified from the $i$-th order sub-graph, where $i=0,1, \ldots, m, j=1,2, \ldots n_{L, i}, m$ is the order of decomposition, and $n_{L, j}$ is number of paths found for the $j$-th subgraph. Also, $B_{i j}$ is the intersection of all the events indicated in the set coefficients identified from the lower-order graphs. The disjoint link sets are therefore identified as $L_{1}=E_{01}, L_{2}=B_{11} \cap E_{11}, \ldots, L_{N_{L}}=B_{m n_{L, m}} E_{m n_{L, m}}$ where $N_{L}=1+\sum_{i=1}^{m} n_{L, i}$.

Although Eq. (1) could be applied to compute the CR between a source node and a terminal node, identifying all the disjoint links for a very large complex network is a challenge. As an alternative, providing the upper and lower bounds of CR could be assessed by identifying as many disjoint links and disjoint cuts as affordable.

The connectivity failure probability could be computed as follows:

$$
F=\sum_{i=1}^{m} \sum_{j=1}^{n_{C, i}} P\left(D_{i j}\right)=\sum_{i=1}^{N_{L}} P\left(C_{i}\right),
$$


where $D_{i j}\left(i=1,2, \ldots, m, j=1,2, \ldots, n_{C, i}\right)$ is the intersection of all the events indicated in the set coefficients to obtain the $j$-th disconnected subgraph among the $i$-th order subgraphs. The disjoint cut sets are therefore identified as $C_{1}=D_{11}, C_{2}=D_{12}, \ldots, C_{N_{C}}=D_{m n_{C, m}}$ where $N_{C}=\sum_{i=1}^{m} n_{C, i}$.

With a sufficient number of disjoint link and cut sets identified, the connectivity reliability and connectivity failure probability can be bounded by

$$
\begin{gathered}
\sum_{i=1}^{n_{L+t}} P\left(L_{i}\right) \leq R \leq 1-\sum_{i=1}^{n_{C \cdot t}} P\left(C_{i}\right), \text { and } \\
\sum_{i=1}^{n_{C+t}} P\left(C_{i}\right) \leq F \leq 1-\sum_{i=1}^{n_{L+1}} P\left(L_{i}\right),
\end{gathered}
$$

where $n_{L, t}$ and $n_{C, t}$ are afforded and identify disjoint link and cut sets.

Once the difference between upper and lower bound is small enough, we can estimate the CR through the following equation,

$$
R \approx \frac{1}{2}\left[\sum_{i=1}^{n_{L t}} P\left(L_{i}\right)+1-\sum_{i=1}^{n_{C, t}} P\left(C_{i}\right)\right]
$$

The above S-RDA can be applied to a network with a single initial node and a single terminal node. But in civil and infrastructure engineering, multi-origin and multi-terminal nodes commonly exist. Hence, this study uses a logical expansion to RDA [50]. To be specific, add a virtual node to the graph and connect the added virtual node with all the initial nodes, as commonly done in network optimization problems [51]. The reliability of the added virtual node is 1.0. The CR between the multi-initial nodes and the terminal nodes is transformed as the CR between the added virtual node and the terminal node, which could be computed with the S-RDA approach. This S-RDA as described is based on a node-centric perspective, in which the model considers only the failure of nodes when computing the CR. The S-RDA can also model the failure of links through transforming a "link" to a "node" in the graph [19].

2.2. Node importance measure based on network connectivity reliability

In infrastructure networks, different components may contribute differently to $\mathrm{CR}$. This paper uses a Risk Achievement Worth (RAW) metric to quantify the importance of a component (node) by computing the $\mathrm{CR}$ decrease (or failure probability increase) of the system while a node is removed from the system. As the removal of an important node will trigger a large CR change, node importance (NI) in terms of CR for the $i$-th node is defined as: 


$$
N I_{R i}=\frac{1-\frac{1}{N_{T}} \sum_{j=1}^{N_{T}} R_{j}^{(i)}}{1-\frac{1}{N_{T}} \sum_{j=1}^{N_{T}} R_{j}},
$$

where $R_{j}$ denotes the $\mathrm{CR}$ of terminal node $j\left(j=1,2, \ldots, N_{T} ; N_{T}\right.$ is the number of terminal nodes), which is defined as the probability that node $j$ is connected to at least one source node; and $R_{j}^{(i)}$ denotes the CR of terminal node $j$ when node $i$ is removed $(i=1,2, \ldots, N)$.

Note that S-RDA and NI metrics are defined based on the assumption that node failures are statistical independence, even though correlated failures and user-defined failure events could also be captured $[52,53]$.

\section{Network controllability}

\subsection{Topological controllability theory}

There are models to capture the controllability of a real system that is driven by linear and time-invariant processes $[9,42]$, such as:

$$
\frac{d x(t)}{d t}=A x(t)+B u(t),
$$

where $x(t)=\left(x_{1}(t), \ldots, x_{\mathrm{N}}(t)\right)^{\mathrm{T}}$ denotes the state of a system with $N$ nodes at time $t$. The $N \times N$ matrix $A$ describes the system's wiring diagram. The $N \times M$ matrix $B$ is the input that identifies the nodes controlled by an outside controller $(M \leqslant N)$. And the system is controlled using a time-dependent input vector $u(t)=\left(u_{1}(t), \ldots, u_{\mathrm{M}}(t)\right)^{\mathrm{T}}$.

In power networks, the dynamics of transient instabilities are non-linear in nature and evolve at fast speeds. However, there is recognition that in practice Direct Current (DC) power flow models [54], as well as decoupled Alternating Current (AC) power flow models or fully coupled AC models, are routinely used for steady-state power dispatch as well as for power system planning [55]. For steady-state regimens, the topological features of real power networks still provide useful insights as a prerequisite for possible nonlinear control via generators' dispatch or even system reconfiguration [56] or transmission switching [57] in the future. Therefore, this study uses the linear dynamics described in Eq. (7) to support our topological controllability investigation. 
One could compute the controllability of linear systems through the Kalman or Hautus criterion [58]. However, they require the assessment of ranks for $2^{N}-1$ distinct combinations, which is computationally prohibitive to establish controllability.

Alternatively, Liu et al. [9] proved that the minimum number of driver nodes needed to maintain full control of a network is determined by the 'maximum matching' in the network, or the maximum set of links that do not share start or end nodes. The maximum matching could be identified through the Hopcroft-Karp (H-K) algorithm [59]. A node is said to be matched if a link in the maximum matching set points at it; otherwise, it is unmatched. One can gain full control over a directed network if and only if there is direct control of each unmatched node and there are directed paths from the input signals to all matched nodes where controlled nodes are the driver nodes. More details of the TC algorithmic notions can be found in Liu et al. [9].

To measure the controllability attributes of a network, this paper defines a controllability index (CI), formulated as follows:

$$
C=\frac{N-N_{D}}{N},
$$

where $N_{D}$ is the minimum number of driver nodes needed to fully control the network.

\subsection{Node importance measure based on network controllability}

It is natural to expect that a more controllable network needs less driver nodes. However, the same minimal number of driver nodes occurs in many combinations, such that the driver node sets also affect the controllability attributes of a network. The more feasible configurations, the easier to control a system in principle. However, it is almost impossible to enumerate all the configurations of driver nodes directly, since the maximum possible combinations number is combinatorial as: $c\left(N, N_{\mathrm{D}}\right)=N ! /\left[N_{\mathrm{D}} !\left(N-N_{\mathrm{D}}\right)\right.$ !] when $N_{\mathrm{D}}$ is fixed, which is impractical for large $N$. To handle the enumeration process, this paper uses an algorithm proposed by Jia et al. [43] as a first step to identify critical, intermittent and redundant nodes as defined in Section 1.

With critical, intermittent and redundant nodes identified, along with the number of critical nodes $\left(N_{C}\right)$, intermittent nodes $\left(N_{I}\right)$ and redundant nodes $\left(N_{R}\right)$, the potential number of combinations of driver node sets decreases significantly. Then, this paper enumerates all the possible combinations of those intermittent nodes within $c\left(N_{I},\left(N_{D}-N_{C}\right)\right)$ steps, as shown in Algorithm 1.

\footnotetext{
Algorithm 1: Quantify number of configurations with minimal driver nodes
} 


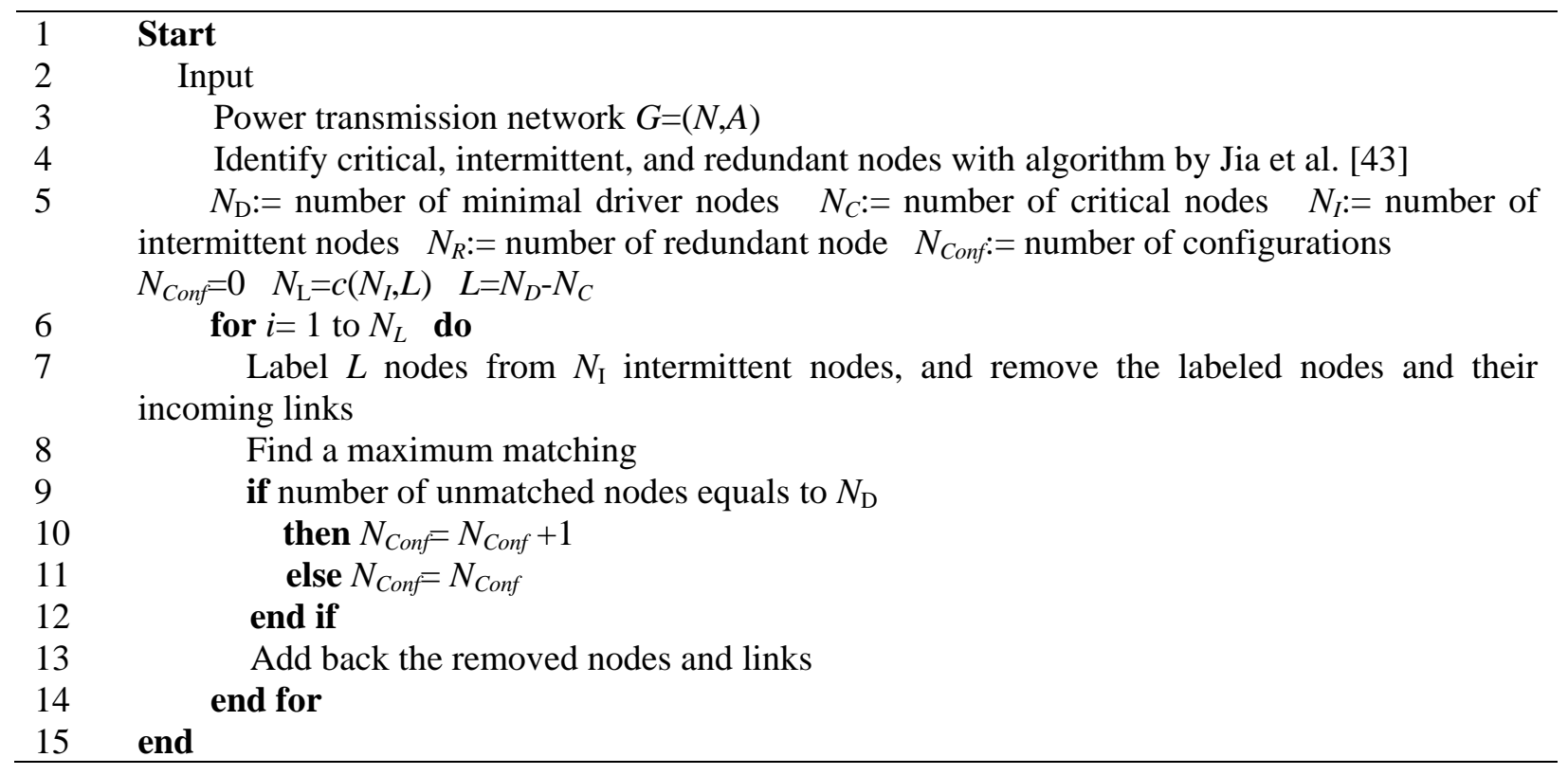

With the above algorithms, the number of combinations of minimal driver node sets can be computed. As a result, this study develops a controllability-based metric to measure NI in terms of TC,

$$
N I_{C i}=a \frac{1-C^{(i)}}{1-C}+(1-a) \log \left(\frac{N_{\text {Conf }}}{N_{\text {Conf }}^{(i)}}\right),
$$

where $C$ is the CI of the network, $C^{(i)}$ is the CI of the network with node $i$ isolated from the network (which means all the edges that connected to node $i$ are removed), and $N_{\text {conf }}$ is the number of controllable configurations with the minimal number of driver nodes, while $N_{\text {conf }}^{(i)}$ is the number of configurations with the minimal number of driver nodes when node $i$ is isolated.

In Eq. (9), two aspects are monitored to quantify the importance of a node, under the assumption that node $i$ is isolated from the network. One is to monitor $C^{(i)}$, and the other is to monitor $N_{\text {conf }}{ }^{(i)}$. For node $i$, if its isolation results in a low $C^{(i)}$ level, the node should be important. This is because more nodes are needed for controlling the system, and as a result the system becomes 'hard' to be controlled with the isolation of the said node. Similarly, if the isolation of a node leads to a low number of controllable configurations $N_{\text {conf }}{ }^{(i)}$, the node should also be important. Because a low $N_{\text {conf }}^{(i)}$ implies a small number of possible control configurations, and this makes the system difficult to control. To balance the relative effect of $C^{(i)}$ and the number of configurations $N_{\text {conf }}{ }^{(i)}$, a weighting coefficient $a$ is applied. Section 6 will provide a discussion on sensitivity to coefficient $a$. 
Given their networked nature, the topological features of infrastructure systems impact system CR and TC properties. However, the effects that link topological features, such as links density and degree heterogeneity have on both CR and TC is not clear today. With the above presented S-RDA (Section 2) and expanded TC theory (Section 3), CR and TC features for different topological graphs can be computed and quantitative comparisons established.

\section{Impact of global topological metrics on CR and TC}

In this paper, eight city-level power transmission grids from cities across five U.S. states that are susceptible to damage from natural hazards are employed. The grids consist of $110 \mathrm{kV}$ to $765 \mathrm{kV}$ transmission-level power lines connecting generators and substations. The raw network data is obtained in GIS format from the "Platts" repository for maps and geospatial data [60]. In the power networks, a node denotes a power plant or a substation, and an edge denotes the power transmission lines between nodes. The network topological details are shown in Table 1, and an example of the spatial extent of a power transmission network (Amarillo, TX) is shown in Fig. 1.

Table 1 Topological properties of the 8 city-level power transmission networks

\begin{tabular}{cccccc}
\hline $\begin{array}{c}\text { City } \\
\text { number }\end{array}$ & City names & $\begin{array}{c}\text { Number } \\
\text { of nodes }\end{array}$ & $\begin{array}{c}\text { Source } \\
\text { nodes }\end{array}$ & $\begin{array}{c}\text { Terminal } \\
\text { nodes }\end{array}$ & $\begin{array}{c}\text { Number } \\
\text { of links }\end{array}$ \\
\hline 1 & Amarillo, TX & 47 & 8 & 39 & 62 \\
2 & Lakeland, FL & 50 & 10 & 40 & 69 \\
3 & El Paso, TX & 52 & 8 & 44 & 65 \\
4 & San Luis Obispo, CA & 57 & 6 & 51 & 69 \\
5 & Bulls Gap, TN & 62 & 6 & 56 & 91 \\
6 & Palm Bay, FL & 66 & 7 & 59 & 98 \\
7 & Greenville, NC & 75 & 2 & 73 & 86 \\
8 & Wilmington, NC & & & & \\
13
\end{tabular}


Fig. 1. Spatial extent of the power transmission network of Amarillo, TX. A source node is a power plant and a terminal node is a substation.

The power transmission network (PTN) of Amarillo, TX, and thousands of artificial networks generated based on the PTN are used as illustrations below (similar calculations performed for other networks). The failure probabilities of each node are established first. The importance of nodes in Amarillo, TX, as a function of different failure probabilities $\{0.001,0.01$, $0.02,0.05,0.1,0.15,0.2\}$, is computed and compared. Results show that different failure probabilities result in different absolute values of node importance, but the node importance rankings and their relative values of element importance remain similar, as shown in Fig. 2 (note this study stresses relative importance rather than absolute importance). As a result, the failure probability $\left(p_{f}\right)$ of each node is assumed to be a moderate value ( 0.1 subsequently) to trigger failures and enable quantification of element removal effects on CR and TC.

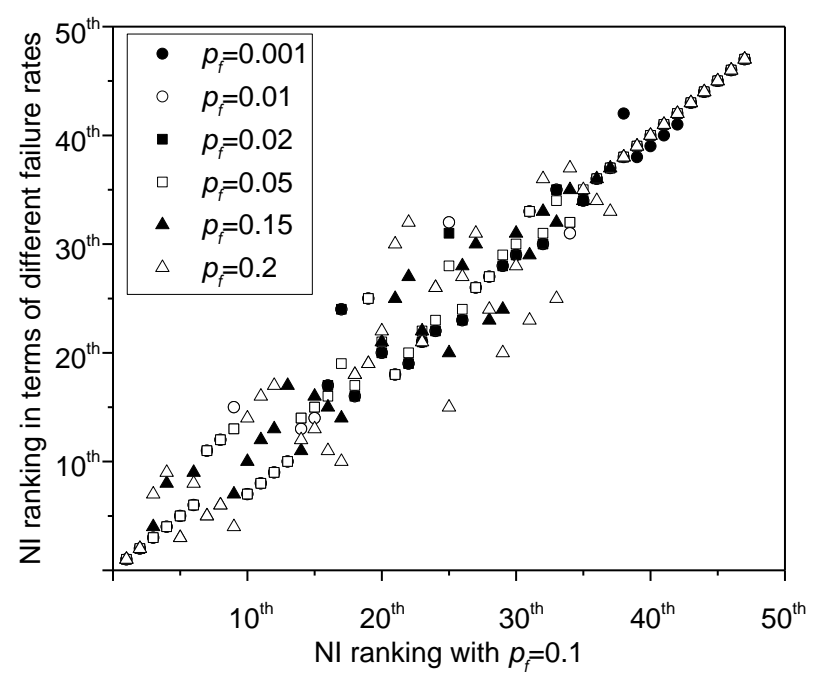

Fig. 2. The importance ranking of nodes in the PTN of Amarillo, TX, in terms of different failure probabilities.

Recall the CR of each terminal node $\left(R_{i}\right)$ is the reliability that the node $i$ is connected to at least one power plant. Across all terminal nodes, this paper calculates the average connectivity reliability (ACR). The minimal driver node set needed to fully control the network is also established, estimating the CI of the networks with Eq. (8).

This paper also computes the ACR and CI of a minimal spanning tree (MST) of the networks, which is generated using Kruskal's algorithm [61]. An MST here is a subset of the 
edges that form a tree that connects every vertex in the graph, where the total weight (line distance) of all the edges in the tree is minimized. MSTs provide idealized reference points.

In order to detect the impact from link density (average degree), artificial networks are generated by adding (or deleting) links randomly to (from) the original PTN while keeping the number of nodes fixed. In the link deletion process, one random link $\left(e_{k}\right)$ is deleted in each step until the link density equals that of the target density. Before deleting each link, we check whether the edge deleting process results in some nodes becoming isolated. If not, the edge $\left(e_{k}\right)$ is removed from the graph; if yes, edge $\left(e_{\mathrm{k}}\right)$ is kept. The average degree ranges from 2.2 to 3.2, which covers most of the link densities of current infrastructure networks [62]. At each average degree level, this paper generates $10^{3}$ networks to capture randomness while affording variability quantification.

Fig. 3 shows the convergence rate in terms of number of simulations for both CR and TC metrics. It can be seen that with about 300 simulations the mean CI converges to the mean value of $10^{3}$ simulations closely. Different from the TC, the mean of the ACR converges more quickly (within 50 simulations). Nevertheless, for both CR and TC, $10^{3}$ samples are enough to converge to a stable value.

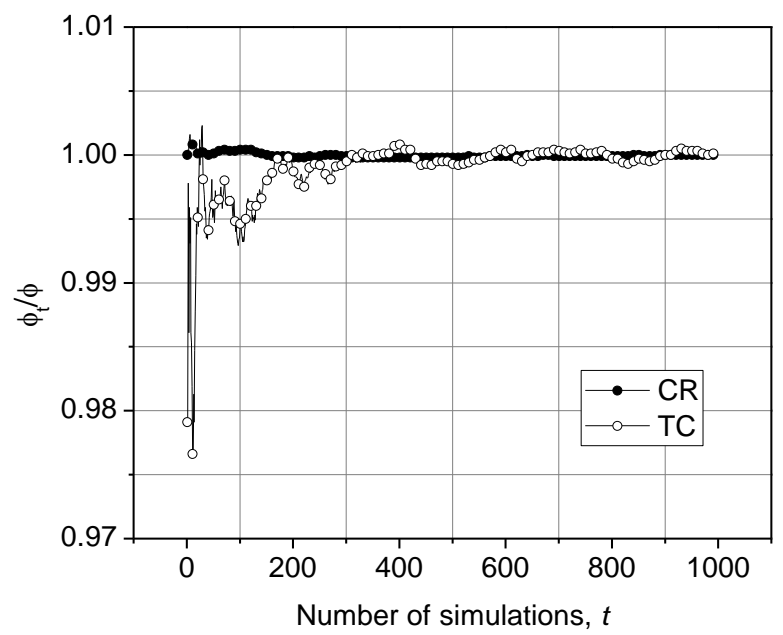

Fig. 3. Rate of convergence in terms of number of simulations. $\phi_{t}$ is the mean of ACR (or CI) of $t$ simulations $\left(t \leq 10^{3}\right)$; and $\phi$ is the mean of ACR (or CI) of the $10^{3}$ simulations for a 2.2 average degree $\langle k>$ version of the Amarillo, TX network.

The mean ACR (MACR) and mean controllability index (MCI) of the networks with different average degree is shown in Fig. 4. Lower bounds for ACR and CI are provided by the 
MST configurations. It can also be seen that as the average degree increases, both MACR and MCI increase with a similar trend.

The correlation between the increasing trend for MACR and MCI (as average degree increases), is quantified using the linear Pearson correlation coefficient [63], giving a value between +1 and -1 inclusive, where 1 is total positive correlation, 0 is no correlation, and -1 is total negative correlation. The Pearson correlation coefficient between MACR and MCI is 0.9796 .

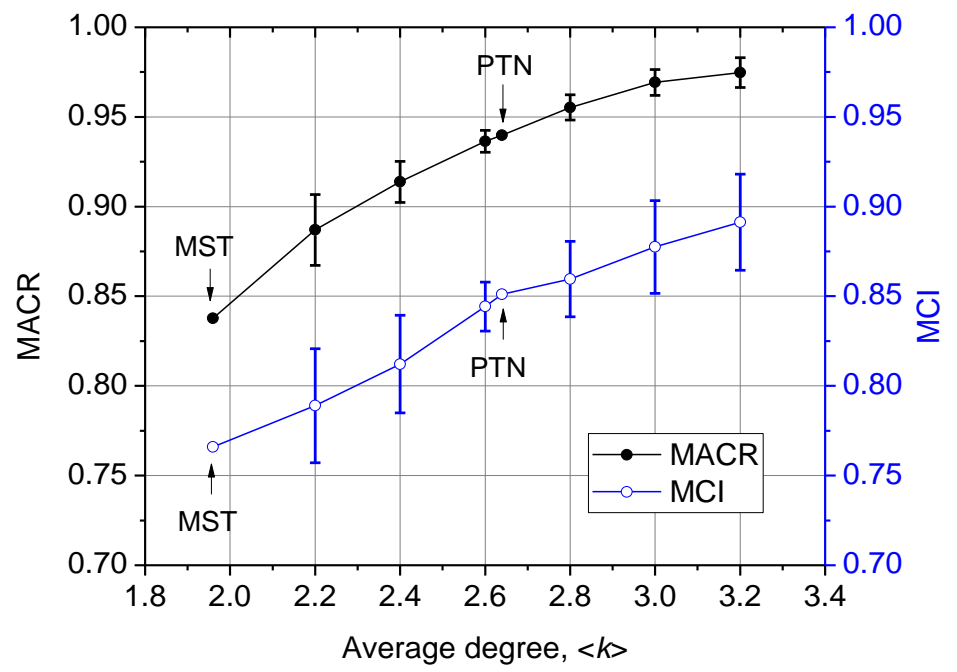

Fig. 4. Mean of the average connectivity reliability (MACR) across network realizations, and mean controllability index (MCI) in terms of different average degree of the networks, $\langle k\rangle$.

To analyze the impacts from degree heterogeneity, a metric that measures it is herewith defined as:

$$
H=\frac{1}{N} \sum_{i=1}^{N}\left(k_{i}-\langle k\rangle\right)^{2},
$$

where $k_{\mathrm{i}}$ is the degree of node $i$ and $\langle k\rangle$ is the average degree of the network. 


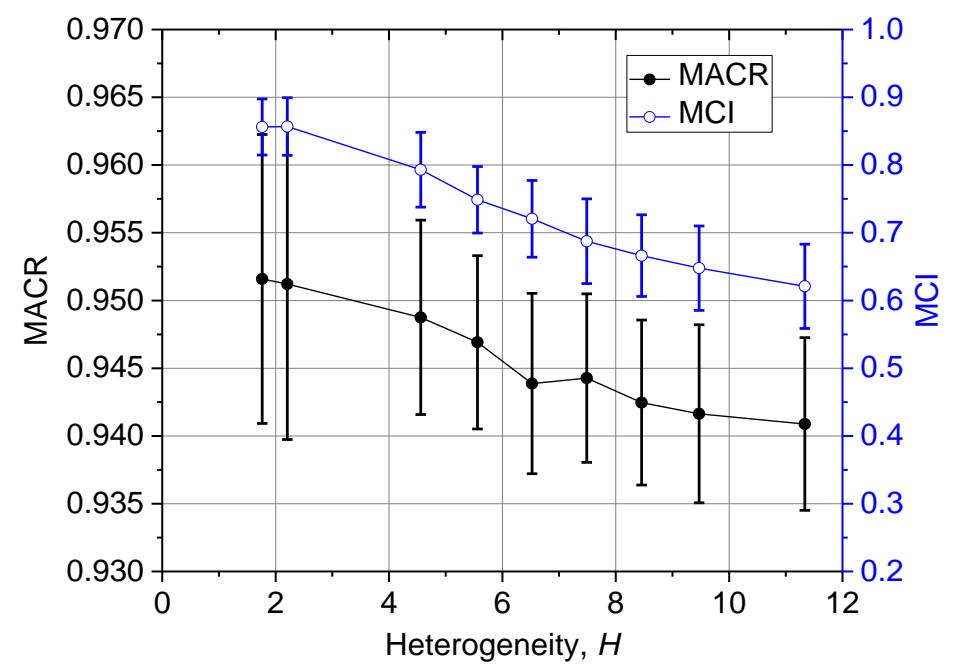

Fig. 5. Mean of the average connectivity reliability (MACR) across networks, and mean controllability index (MCI) in terms of degree heterogeneity, $H$.

This paper generates $10^{3}$ Erdos-Renyi (E-R) random networks [64], which obey a Poisson degree distribution (when $N>>1$ ), as well as $10^{3}$ Scale-free networks [65], whose degree distribution follow power laws. To avert the impacts from link density and network scale on CR and TC, the number of nodes and links are preserved as the networks are generated. Fig. 5 presents the MACR and MCI of these generated networks in terms of different degreeheterogeneity $H$ levels. It can be seen that as $H$ increases, both MACR and MCI decrease. The Pearson correlation coefficient between the two curves is 0.9903 . In other words, the CR and TC match with each other highly in terms of degree heterogeneity and average degree levels.

We also observe that in Fig. 5 the error bar of MACR is much larger than that of MCI, while in Fig. 4 the error bar of MACR is much smaller than that of MCI. This indicates that the average degree's impact on CR is more direct than on TC while degree heterogeneity's impact on $\mathrm{CR}$ is not as significant as that on TC (see scales too).

Adding a link to a network (which does not change the degree heterogeneity much) will increase the reliability of certain nodes, and as a result, will improve the CR of the whole network. However, adding a link to the network does not guarantee that controllability will improve. For example, adding a link between two nodes that are not only a matched node but also a parent node of another matched node, the number of driver nodes will not change, and either does the CI. Hence, CR is more consistent with the average degree change than TC. However, if the average degree is fixed for a network, but its degree becomes heterogeneous, it means that the proportion of high degree and low degree nodes increases while medium degree 
node proportion reduces. In this case, the low degree nodes tend to be unmatched since they have few incoming links, while the high degree nodes tend to be always matched since they have many incoming links. This makes the CI of the network decrease significantly. As for CR, a high proportion of high degree nodes increases network $\mathrm{CR}$, but a high proportion of low degree nodes decreases network CR. The offset effect results in that CR is not too sensitive to degree heterogeneity.

Summing previous results, a dense and homogeneous network is better than a sparse and heterogeneous network to excel under both CR and TC considerations - a key insight for smart infrastructure network design in the future. This finding could guide the improvement of existing infrastructure networks, which tend to be sparse, and, depending of the system, possess various degrees of heterogeneity. Other factors should also be taken into consideration in practical

engineering design, including resilience, costs, functionality ratings, etc. Particularly, the robustness that contributes to resilience is one of the fundamental aspects of infrastructure networks, which is discussed next.

\section{CR-based and TC-based network robustness}

Besides topological effects, critical infrastructure systems may be subjected to random failures and target disruptions during normal operation and adversarial times. For targeted disruptions, attacking high degree or high betweenness nodes would be efficacious [66]. Therefore, this section assesses networked infrastructure system robustness under random failures, degree-based attack and betweenness-based removals, as follows:

Random failures: In one step, one node fails uniformly at random. Once a node fails, all the edges that are connected to it are removed from the graph;

Degree-based attack: In one step, the node that has the largest degree $\left(k_{\max }\right)$ of the current graph, is removed; if there is more than one node with $k_{\max }$, select the node whose neighbors have the largest degree; for nodes tied here, select the node whose neighbors' neighbors have the largest degree, and so on. All the edges connecting this node will be removed from the graph. In the next step, another node is removed according to the previous rules; and

Betweenness-based attack: The betweenness centrality of node $i\left(b_{i}\right)$ is the number of shortest paths from all nodes (except $i$ ) to all other nodes that pass through node $i$ [67]. Hence, in one step, the node with the highest betweenness centrality $\left(b_{\max }\right)$ of the current graph, is 
removed; if there is more than one node with $b_{\max }$, select the node whose neighbors have the highest betweenness. For nodes tied here, select the node whose neighbors' neighbors have the highest betweenness, and so on; and remove the edges connecting the selected node. In the next step, another node is removed according to the previous rules.

The previous strategies' effects on the MACR and MCI of the PTN of Amarillo, TX are shown in Fig. 6. As the node failures (or removals) increase, the MACR and MCI decrease (albeit at a higher rate for MACR). Their decrease rate is slower under random failures compared to degree-based and betweenness-based removals, while the degree-based and betweennessbased attack have similar impact on each of the MACR and MCI, especially when only few nodes are compromised. Trends are consistent as under degree-based and betweenness-based attack strategies, more edges or critical edges are removed in a single step compared to random failures, and as a result, the CR and CI decrease more rapidly, while targeted removals, especially degree-based end up being more efficacious given their impact on average degree and heterogeneity. The other city-level power transmission networks have similar behavior. The collective results show that $\mathrm{CR}$ and TC remain correlated.

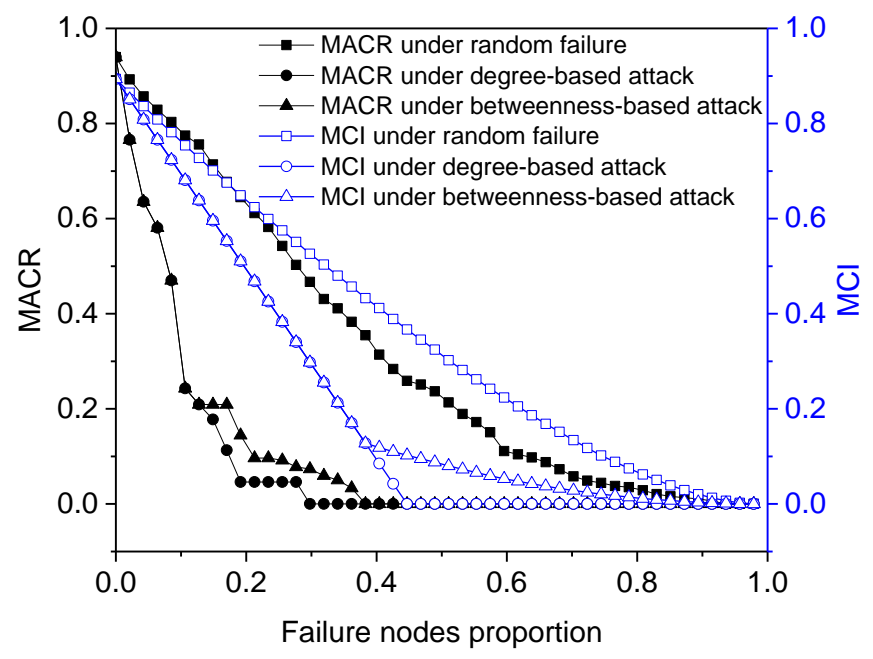

Fig. 6. MACR and MCI of the power transmission network of Amarillo, TX under different failure or attack strategies.

Although degree-based and betweenness-based removals are more effective than random failures degrading the CR and TC metrics, their impact rates need additional observation. This study defines two metrics called relative connectivity reliability decrease (RCRD) and relative controllability index decrease (RCID) to quantify how CR and CI decrease under the degree- 
based and betweenness-based attack strategies compared to random failures. RCRD is computed as $\left(C R_{k}{ }^{d}-C R_{0}\right) /\left(C R_{k}{ }^{r}-C R_{0}\right)$ and $\left(C R_{k}{ }^{b}-C R_{0}\right) /\left(C R_{k}{ }^{r}-C R_{0}\right)$ for degree-based and betweenness-based attack respectively, while RCID is computed with $\left(C_{k}{ }^{d}-C_{0}\right) /\left(C_{k}{ }^{r}-C_{0}\right)$ and $\left(C_{k}^{b}-C_{0}\right) /\left(C_{k}{ }^{r}-C_{0}\right) . C R_{0}$ and $C_{0}$ are the connectivity reliability (i.e. the average $\mathrm{CR}$ of all the terminal nodes) and controllability index of the intact infrastructure networks, respectively; $C R_{k}{ }^{r}$ and $C_{k}{ }^{r}$ are the connectivity reliability and controllability index under random failures with $k$ nodes removed, respectively; $C R_{k}{ }^{d}$ and $C_{k}{ }^{d}$ are the connectivity reliability and controllability index under degreebased attack with $k$ nodes removed, respectively; and $C R_{k}^{b}$ and $C_{k}{ }^{b}$ are the connectivity reliability and controllability index under betweenness-based attack with $k$ nodes removed, respectively.
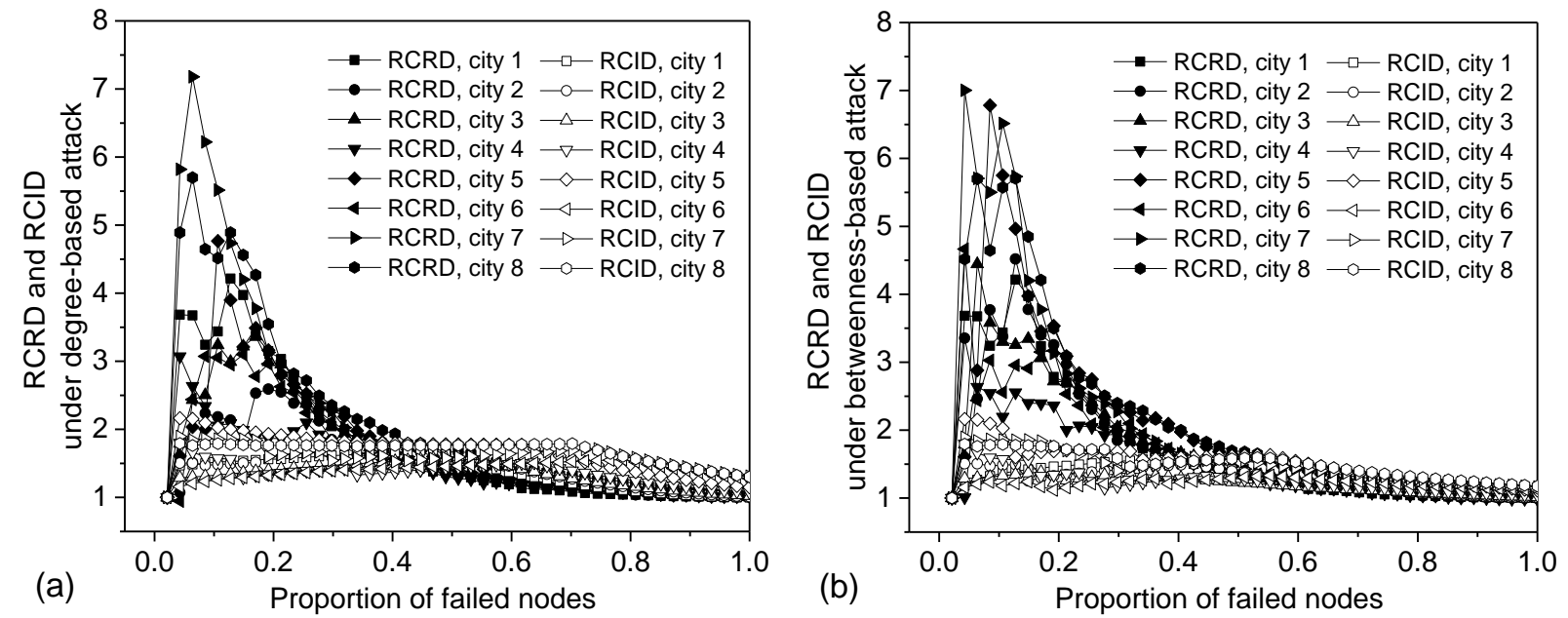

Fig. 7. Relative connectivity reliability decrease (RCRD) and relative controllability index decrease (RCID) under degree-based attack (a) and betweenness-based attack (b).

The RCRD and RCID values are plotted in Fig. 7. It is found that when a few nodes are compromised, RCRD is much larger than RCID under both degree-based and betweenness-based attacks. In other words, CR is more sensitive than CI to degree-based and betweenness-based failures. Recall that in Section 4, it is found that the average degree's impact on CR is more significant than that on TC. As a result, when more edges are moved in each step (when a few nodes are compromised under degree-based or betweenness-based attack), CR decreases significantly, especially at low failure levels, thus highlighting infrastructure investment prioritization in practice. 


\section{Node importance assessment for intervention prioritization}

Before computing and analyzing node importance in terms of CR and TC, Eq. (9) requires assessment of coefficients $a$. The change of number of minimal driver nodes should has larger impact on the controllability attribute of the networks than that of number of configurations, and thus the value of $a$ should be larger than 0.5. Fig. 8 (a) shows the node importance in terms of different coefficient value $a=\{0.6,0.7,0.8,0.9,1.0\}$ for all the nodes of the PTN of Amarillo, TX. One can see that despite variation in $a$, node importance curves have similar trends. Variation of coefficient $a$ changes the exact value of node importance of all the nodes, but has limited impact on the relative value of node importance (or ranking). To highlight this, Fig. 8 (b) plots the TC-based node importance rankings with $a \neq 0.8$ versus $a=0.8$. Most high ranking nodes with $a \neq 0.8$ also ranks high with $a=0.8$, and so do low ranking nodes. Hence, $a$ is fixed at a value of $a=0.8$ in this paper. The node importance in terms of CR and TC can thus be computed with Eq. (6) and Eq. (9).

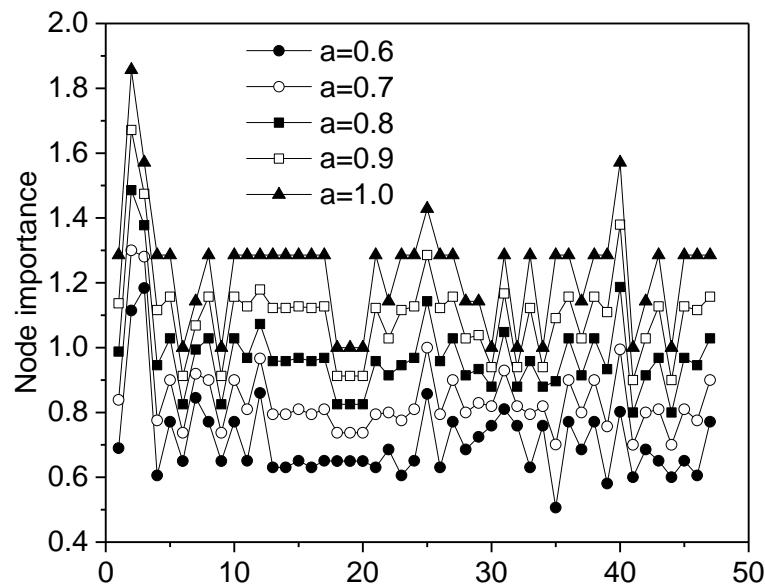

(a)

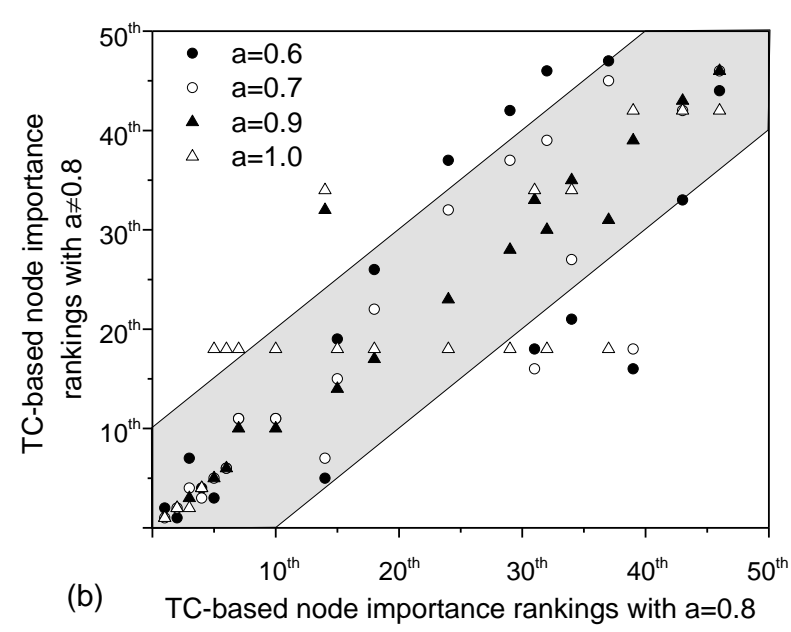

Fig. 8. (a) Node importance based on Eq. (10) of all the nodes of the Amarillo, TX power transmission network in terms of different coefficients $a$; (b) Comparison of TC-based importance ranking between $a \neq 0.8$ and $a=0.8$.

Fig. 9 shows the mean node importance in terms of $\mathrm{CR}\left(M N I_{R}\right)$ and the mean node importance in terms of TC $\left(M N I_{C}\right)$. High degree nodes tend to have higher importance in terms of both CR and TC. The Pearson correlation coefficient between the two curves is 0.9772 . 


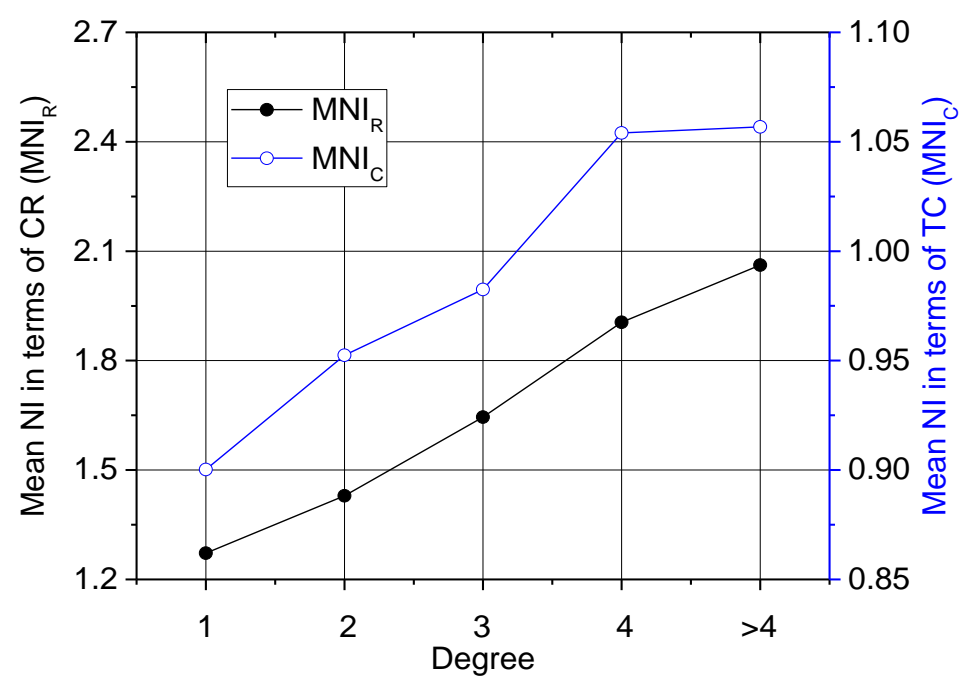

Fig. 9. Mean NI of different degree nodes in terms of CR and TC.

Results so far beg exploration of individual nodes' importance. Each node has two rankings, CR-based and TC-based ranking ( $y$-axis and $x$-axis in Fig. 10 in descending order, respectively). The nodes are divided into two categories: substations and generators. For most of the substation nodes, high ranking in terms of TC corresponds to high ranking in terms of CR (correlation coefficient of 0.7951 ), highlighting that utility practices to upkeep CR could support TC. However, for generator nodes, there is no strong correlation between TC rankings and CR rankings (correlation coefficient of 0.4371). Reasons include the following. When isolating a generator, clearly $\mathrm{CR}$ decreases especially with the degree of the isolated generator. But given the intrinsic CI computations, the CI may remain the same regardless of the degree of an isolated generator. The only change is in the number of controllable configurations with the minimal driver nodes set, but this number is not as important as CI changes, and generator degree's impact remains weak. Hence, no strong correlation between TC rankings and CR rankings is observed within the scope of this paper.

These preliminary findings show that reliability-based measures can identify and inform operators about important nodes, not only for failure prevention but also for future controllability. Elements identified as critical for reliability-based system design, maintenance and restoration may support the deployment of smart technologies that enable controllability and reconfigurability, which in turn support reliability - such feedbacks are yet to be studied in research and practice of smart infrastructure systems [68]. Also note that the reliability-based measure shows some mismatches with controllability-based measure especially regarding power plants, which require future nonlinear functional analyses. 

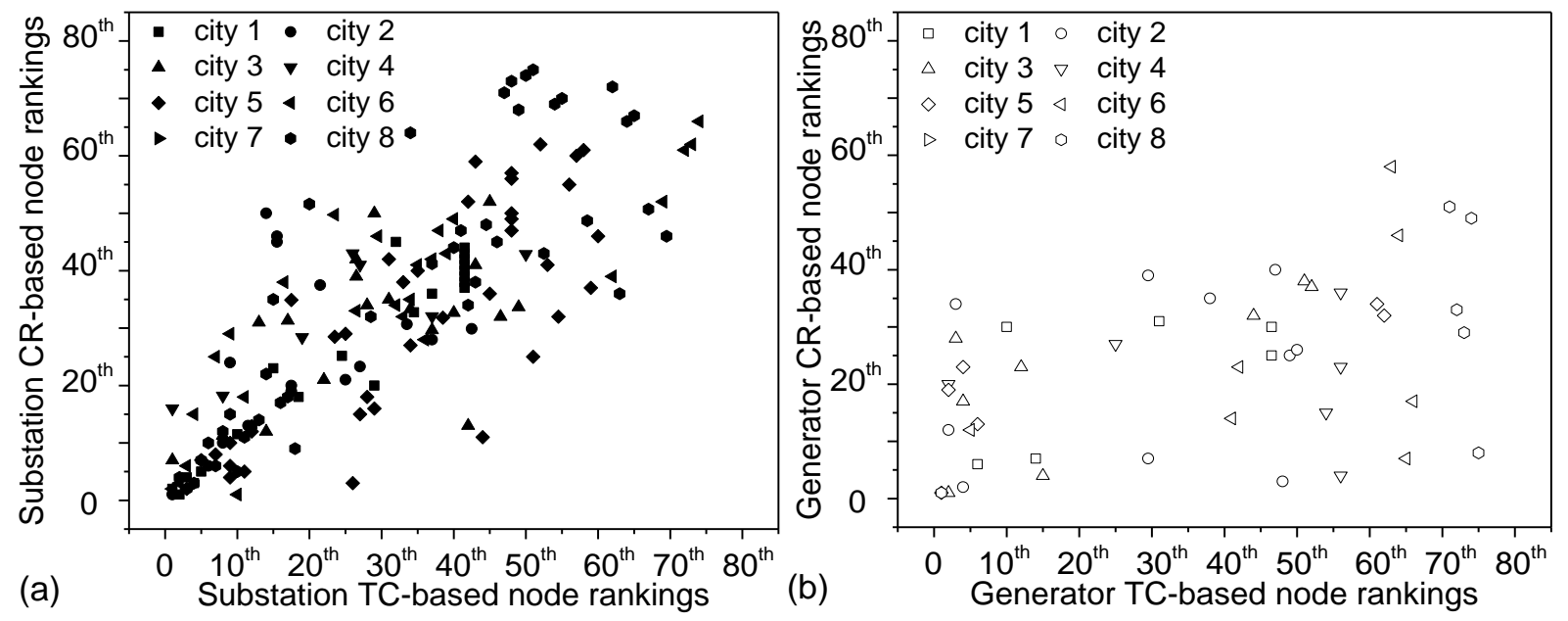

Fig. 10. Correlation between node importance rankings in terms of CR and TC: (a) Substation nodes; (b) Generator nodes.

\section{Conclusions}

Reliability-based design, maintenance, and optimization principles for infrastructure systems continue advancing in engineering practice, but it is unclear whether and how these principles support emerging topological controllability (TC) requirements in the context of smart systems. This paper takes a preliminary step to evaluate the correlation between connectivity reliability (CR) and topological controllability (TC).

For evaluating both CR and TC, this paper develops metrics to quantify them, along with associated node importance (NI) metrics. Particularly for TC, this paper proposes a metric called Controllability Index (CI), and a NI metric that considers both the number of minimal driver nodes and the number of configurations with minimal number of drive nodes.

Taking eight city-level power transmission networks (PTNs) and thousands of artificial networks as examples (which are generated based on the original PTNs), this paper investigates how CR and TC support each other's goals in terms of link density, node degree heterogeneity, and robustness (against failures), as well as node importance (NI).

Results reveal that as the network link density increases or degree heterogeneity decreases (while preserving link density), both the average connectivity reliability (MACR) and mean controllability index (MCI) across system realizations increase. It is also observed that for both $\mathrm{CR}$ and TC, the infrastructure networks are more robust to random failures compared to degreeand betweenness-based removals. In addition, this paper performs a sensitivity analysis of node 
importance, and finds that NI increases with degree (consistent with the network-level MACR and MCI trends).

However, it is found that the average degree's impact on CR is more significant than on TC, while degree heterogeneity's impact is not as significant as that on TC. Numerical simulations also show that when a few nodes are compromised, the relative connectivity reliability decrease (RCRD) is much larger than the relative controllability index decrease (RCID), independent of the degree- or betweenness-based nature of the attacks. As for NI, the CR-based NI rankings show some mismatches with the TC-based measure, particularly for the individual generator nodes, although the CR-based node importance matches well the TC-based measures for most of the substation nodes, highlighting the need for future electrically functional analyses beyond CR.

Also, as this paper focuses on the controllability of networks from a topological perspective, which implies linear dynamics, future work needs to address dynamic-based nonlinear controllability, especially when systems become more interdependent.

\section{Acknowledgments}

This work is funded in part by the U.S. Department of Defense and its Army Research Office through the MURI grant W911NF-13-1-0340, and the U.S. National Science Foundation through grant 1435845 . The present work is also financially supported by the National Natural Science Foundation of China (NSFC) under grants 51534008 and 51576212 and the Fundamental Research Funds for Central Universities in China. The authors also thank Mr. Andres Gonzalez at Rice University for providing S-RDA support.

\section{References}

[1] Ching J, Hsu W-C. An Efficient Method for Evaluating Origin-Destination Connectivity Reliability of Real-World Lifeline Networks. Comput Civ Infrastruct Eng 2007;22:584-96. doi:10.1111/j.1467-8667.2007.00501.x.

[2] Johansson J, Hassel H, Zio E. Reliability and vulnerability analyses of critical infrastructures: Comparing two approaches in the context of power systems. Reliab Eng Syst Saf 2013;120:27-38. doi:10.1016/j.ress.2013.02.027.

[3] Akiyama M, Frangopol DM, Suzuki M. Integration of the effects of airborne chlorides into reliability-based durability design of reinforced concrete structures in a marine environment. Struct Infrastruct Eng 2012;8:125-34.

[4] Jensen HA, Muñoz A, Papadimitriou C, Millas E. Model-reduction techniques for reliability-based design problems of complex structural systems. Reliab Eng Syst Saf 2016;149:204-17. doi:10.1016/j.ress.2016.01.003.

[5] Elshqeirat B, Soh S, Rai S, Lazarescu M. Topology design with minimal cost subject to network 
reliability constraint. IEEE Trans Reliab 2015;64:118-31. doi:10.1109/TR.2014.2338253.

[6] Frangopol D, Liu M. Maintenance and management of civil infrastructure based on condition, safety, optimization, and life-cycle cost. Struct Infrastruct Eng 2007;3:29-41. doi:10.1080/15732470500253164.

[7] Yeh W-C, Lin Y-C, Chung YY, Chih M. A Particle Swarm Optimization Approach Based on Monte Carlo Simulation for Solving the Complex. IEEE Trans Reli 2010;59:212-21.

[8] Mo H, Xie M, Levitin G. Optimal resource distribution between protection and redundancy considering the time and uncertainties of attacks. Eur J Oper Res 2015;243:200-10. doi:10.1016/j.ejor.2014.12.006.

[9] Liu Y-Y, Slotine J-J, Barabási A-L. Controllability of complex networks. Nature 2011;473:16773. doi:10.1038/nature10011.

[10] Kamat SJ, Riley MW. Determination of Reliability Using Event-Based Monte Carlo Simulation. IEEE Trans Reliab 1975;R-24:73-5.

[11] Hwang CL, Tillman FA, Lee MH. System-Reliability Evaluation Techniques for Complex/Large System: A Review. IEEE Trans Reliab 1981;R-30:416-23.

[12] Adachi T, Ellingwood BR. Serviceability of earthquake-damaged water systems: Effects of electrical power availability and power backup systems on system vulnerability. Reliab Eng Syst Saf 2008;93:78-88. doi:10.1016/j.ress.2006.10.014.

[13] Beck JL, Au S-K. Bayesian Updating of Structural Models and Reliability using Markov Chain Monte Carlo Simulation. J Eng Mech 2002;128:380-91. doi:10.1061/(ASCE)07339399(2002)128:4(380).

[14] Song S, Lu Z, Qiao H. Subset simulation for structural reliability sensitivity analysis. Reliab Eng Syst Saf 2009;94:658-65. doi:10.1016/j.ress.2008.07.006.

[15] Ramirez-marquez JE, Coit DW. A Monte-Carlo simulation approach for approximating multi-state two-terminal reliability. Reliab Eng Syst Saf 2005;87:253-64. doi:10.1016/j.ress.2004.05.002.

[16] Dawson R, Hall J. Adaptive importance sampling for risk analysis of complex infrastructure systems. Proc R Soc A Math Phys Eng Sci 2006;462:3343-62.

[17] Iman RL. Latin hypercube sampling. Wiley Online Library; 2008.

[18] Shields MD, Zhang J. The generalization of Latin hypercube sampling. Reliab Eng Syst Saf 2016;148:96-108. doi:10.1016/j.ress.2015.12.002.

[19] Lim H, Song J. Efficient risk assessment of lifeline networks under spatially correlated ground motions using selective recursive decomposition algorithm. Earthq Eng Struct Dyn 2012;41:186182. doi:10.1002/eqe.2162.

[20] Yeh WC. An improved sum-of-disjoint-products technique for the symbolic network reliability analysis with known minimal paths. Reliab Eng Syst Saf 2007;92:260-8. doi:10.1016/j.ress.2005.12.006.

[21] Lee C-Y. Representation of Switching Circuits by Binary-Decision Programs. Bell Syst Tech J 1959;38:985-99.

[22] Bryant RE, Meinel C. Ordered binary decision diagrams. Springer US; 2002.

[23] Dotson W, Gobien J. A new analysis technique for probabilistic graphs. Circuits Syst IEEE Trans 1979;26:855-65.

[24] Li J, He J. A recursive decomposition algorithm for network seismic reliability evaluation. Earthq Eng Struct Dyn 2002;31:1525-39. doi:10.1002/eqe.174. 
[25] Kang W-H, Song J, Gardoni P. Matrix-based system reliability method and applications to bridge networks. Reliab Eng Syst Saf 2008;93:1584-93. doi:10.1016/j.ress.2008.02.011.

[26] Yeh WC. A simple universal generating function method to search for all minimal paths in networks. IEEE Trans Syst Man, Cybern Part ASystems Humans 2009;39:1247-54. doi:10.1109/TSMCA.2009.2026209.

[27] Dueñas-Osorio L, Rojo J. Reliability Assessment of Lifeline Systems with Radial Topology. Comput Civ Infrastruct Eng 2011;26:111-28. doi:10.1111/j.1467-8667.2010.00661.x.

[28] Chang Y, Mori Y. A study on the relaxed linear programming bounds method for system reliability. Struct Saf 2013;41:64-72. doi:10.1016/j.strusafe.2012.11.002.

[29] Kang W, Song J. Evaluation of multivariate normal integrals for general systems by sequential compounding. Struct Saf 2010;32:35-41. doi:10.1016/j.strusafe.2009.06.001.

[30] Kang WH, Kliese A. A rapid reliability estimation method for directed acyclic lifeline networks with statistically dependent components. Reliab Eng Syst Saf 2014;124:81-91. doi:10.1016/j.ress.2013.11.015.

[31] Provan JS, Ball MO. Computing Network Reliability in Time Polynomial in the Number of Cuts. Oper Res 1984;32:516-26.

[32] Feng G, Patelli E, Beer M, Coolen FPA. Imprecise system reliability and component importance based on survival signature. Reliab Eng Syst Saf 2016;150:116-25. doi:10.1016/j.ress.2016.01.019.

[33] He L, Zhang X. Fuzzy reliability analysis using cellular automata for network systems. Inf Sci (Ny) 2016:1-15. doi:10.1016/j.ins.2016.01.102.

[34] Hosseini SA, Wadbro E. Connectivity reliability in uncertain networks with stability analysis. Expert Syst Appl 2016;57:337-44. doi:10.1016/j.eswa.2016.03.040.

[35] Bistouni F, Jahanshahi M. Evaluating failure rate of fault-tolerant multistage interconnection networks using Weibull life distribution. Reliab Eng Syst Saf 2015;144:128-46. doi:10.1016/j.ress.2015.07.023.

[36] Jafary B, Fiondella L. A universal generating function-based multi-state system performance model subject to correlated failures. Reliab Eng Syst Saf 2016;152:16-27. doi:10.1016/j.ress.2016.02.004.

[37] Johari A, lari AM. System reliability analysis of rock wedge stability considering correlated failure modes using sequential compounding method. Int J Rock Mech Min Sci 2016;82:61-70. doi:10.1016/j.ijrmms.2015.12.002.

[38] Fan W, Yang P, Ang AH, Li Z. Analysis of complex system reliability with correlated random vectors. Probabilistic Eng Mech 2016;45:61-9. doi:10.1016/j.probengmech.2016.03.004.

[39] Liu W, Li J. An improved recursive decomposition algorithm for reliability evaluation of lifeline networks. Earthq Eng Eng Vib 2009;8:409-19.

[40] Liu W, Li J. An improved cut-based recursive decomposition algorithm for reliability analysis of networks. Earthq Eng Eng Vib 2012;11:1-10.

[41] Lim H-W, Song J, Kurtz N. Seismic reliability assessment of lifeline networks using clusteringbased multi-scale approach. Earthq Eng Struct Dyn 2014:n/a - n/a. doi:10.1002/eqe.2472.

[42] Lombardi A, Hörnquist M. Controllability analysis of networks. Phys Rev E 2007;75:056110. doi:10.1103/PhysRevE.75.056110.

[43] Jia T, Liu Y-Y, Csóka E, Pósfai M, Slotine J-J, Barabási A-L. Emergence of bimodality in controlling complex networks. Nat Commun 2013;4:1-6. doi:10.1038/ncomms3002. 
[44] Diao K, Rauch W. Controllability analysis as a pre-selection method for sensor placement in water distribution systems. Water Res 2013;47:6097-108. doi:10.1016/j.watres.2013.07.026.

[45] Dekker AH. Simulating network robustness for critical infrastructure networks. Conf Res Pract Inf Technol Ser 2005;38:59-68.

[46] Yan G, Ren J, Lai Y-C, Lai C-H, Li B. Controlling Complex Networks: How Much Energy Is Needed? Phys Rev Lett 2012;108:218703. doi:10.1103/PhysRevLett.108.218703.

[47] Wang B, Gao L, Gao Y, Deng Y. Maintain the structural controllability under malicious attacks on directed networks. EPL (Europhysics Lett 2013;101:58003. doi:10.1209/0295-5075/101/58003.

[48] Ding J, Lu Y-Z. Control backbone: An index for quantifying a node's importance for the network controllability. Neurocomputing 2015;153:309-18. doi:10.1016/j.neucom.2014.11.024.

[49] Liu Y, Slotine J, Barabási A. Control centrality and hierarchical structure in complex networks. PLoS One 2012;7:1-8. doi:10.1371/Citation.

[50] Kim Y, Kang W-H. Network reliability analysis of complex systems using a non-simulation-based method. Reliab Eng Syst Saf 2013;110:80-8. doi:10.1016/j.ress.2012.09.012.

[51] Ahuja R, Magnanti T, Orlin J. Network Flows: Theory, Algorithms, and Applications. Prentice Hall; 1993.

[52] Song J, Kang W-H. System reliability and sensitivity under statistical dependence by matrix-based system reliability method. Struct Saf 2009;31:148-56. doi:10.1016/j.strusafe.2008.06.012.

[53] Dueñas-Osorio L, Padgett JE. Seismic Reliability Assessment of Bridges with User-Defined System Failure Events. J Eng Mech 2011;137:680-90. doi:10.1061/(ASCE)EM.19437889.0000272.

[54] Eremia, Mircea; Shahidehpour M. Handbook of Electrical Power System Dynamics. Wiley Online Library; 2013.

[55] Von Meier A. Electric power systems: a conceptual introduction. John Wiley \& Sons; 2006.

[56] Amanulla B, Chakrabarti S, Singh SN. Reconfiguration of power distribution systems considering reliability and power loss. IEEE Trans Power Deliv 2012;27:918-26. doi:10.1109/TPWRD.2011.2179950.

[57] Hedman KW, O’Neill RP, Fisher EB, Oren SS. Optimal transmission switching with contingency analysis. IEEE Trans Power Syst 2009;24:1577-86. doi:10.1109/TPWRS.2009.2020530.

[58] Yuan Z, Zhao C, Di Z, Wang W-X, Lai Y-C. Exact controllability of complex networks. Nat Commun 2013;4:2447. doi:10.1038/ncomms3447.

[59] Hopcroft JE, Karp RM. An $n^{\wedge} 5 / 2$ algorithm for maximum matchings in bipartite graphs. SIAM J Comput 1973;2:225-31.

[60] Platts. http://www.platts.com/products/gis-data 2013.

[61] Buhl J, Gautrais J, Reeves N, Solé R V., Valverde S, Kuntz P, et al. Topological patterns in street networks of self-organized urban settlements. Eur Phys J B 2006;49:513-22. doi:10.1140/epjb/e2006-00085-1.

[62] Li J, Dueñas-Osorio L, Chen C, Berryhill B, Yazdani A. Characterizing the Topological and Controllability Features of U.S. Power Transmission Networks. Phys A Stat Mech Its Appl 2015:Under review.

[63] Ang AHS, Tang WH. Probability concepts in engineering. 2004.

[64] Erdős P, Rényi A. On the evolution of random graphs. Publ Tke Matkemafical Insfifufe Hungarian Acad Sci 1960;5:343-7. 
[65] Barabási AL, Albert R. Emergence of Scaling in Random Networks. Science (80- ) 1999;286:50912. doi:10.1126/science.286.5439.509.

[66] Huang X, Gao J, Buldyrev S V., Havlin S, Stanley HE. Robustness of interdependent networks under targeted attack. Phys Rev E 2011;83:065101. doi:10.1103/PhysRevE.83.065101.

[67] Lewis TG. Network science: Theory and applications. John Wiley \& Sons; 2011.

[68] Poyrazoglu G, Oh H. Optimal Topology Control With Physical Power Flow Constraints and N-1 Contingency Criterion. IEEE Trans Power Syst 2015;30:3063-71. 